\title{
Sublingual immunotherapy in mite-sensitized patients with atopic dermatitis: a randomized controlled study
}

\author{
Nansheng $\mathrm{Yu}^{1}$, Hongyu Luo ${ }^{2}$, Donglong Liang ${ }^{1}, \mathrm{Na} \mathrm{Lu}^{1}$ \\ ${ }^{1}$ Department of Dermatology, Affiliated Shunde Hospital of Guangzhou Medical University, China \\ ${ }^{2}$ Department of Dermatology, The Fifth Affiliated Hospital of Southern Medical University, China
}

Adv Dermatol Allergol 2021; XXXVIII (1): 69-74

DOI: https://doi.org/10.5114/ada.2021.104281

\begin{abstract}
Introduction: Sublingual immunotherapy (SLIT) has been shown to be efficacious in patients with airway allergic diseases. However, less data have been demonstrated to show the efficacy of SLIT in patients with atopic dermatitis (AD). Aim: This study is to evaluate, in a randomized controlled study, the effect of SLIT with house dust mite (HDM) in patients with mild-moderate AD.

Material and methods: AD patients aged 4 to 60 years with a Scoring Atopic Dermatitis (SCORAD) score of 7-40 and sensitization to HDM were enrolled in the study. SLIT or control treatment was given for 24 months. SCORAD, visual analog scale (VAS) score were recorded at 6, 12, 24 month, and rescue medications were required to be recorded in the diary card. A serum level of specific IgE was tested at 24-month treatment.

Results: Ninety-six patients were enrolled, and forty-eight were allocated to SLIT. Thirty-nine patients in the SLIT group and thirty-eight patients in the control group completed the study. The patients in the SLIT group had significantly decreased $\triangle$ SCORAD, VAS and rescue medication score from 12 months' treatment compared with the control group $(p<0.05)$. At 24 months of treatment, no significant change of specific IgE $(p<0.05)$ was observed in both groups. No severe adverse events were reported during the treatment.

Conclusions: Two years' SLIT to HDM significantly improved the clinical symptoms and reduced drug use in patients with mild-moderate AD. SLIT may represent an additional therapeutic tool for the treatment of AD in properly selected patients.
\end{abstract}

Key words: sublingual immunotherapy, atopic dermatitis, house dust mite.

\section{Introduction}

Atopic dermatitis (AD) is a chronic inflammatory itching skin disease. Although the incidence of $A D$ is relatively high worldwide, affecting a large number of adults and children, the cause of its onset is not particularly clear [1, 2]. The occurrence of AD may be related to both genetic and environmental factors. Although AD is not considered to be a typical allergic disease, allergens, especially house dust mites (HDM) that are easily exposed to in daily life, may promote the development of AD. Moreover, many patients have elevated IgE, which is also thought to be associated with the onset of $\mathrm{AD}[3]$.

Allergen-specific immunotherapy (SIT) is a causal treatment, in addition to improving symptoms, it is thought to slow down the natural progression of the allergy march [4]. Sublingual immunotherapy (SLIT) and subcutaneous immunotherapy (SCIT) are two most commonly used methods in
AIT. HDM SCIT has recently been shown to improve eczema symptoms in $\mathrm{AD}$ patients in a randomized, double-blind and non-controlled trial $[5,6]$. SLIT is considered to be a more convenient and safe treatment alternative to SCIT [7]. However, SLIT has so far been poorly used in the treatment of AD, leaving little clinical evidence of its use in AD patients [8-10].

\section{Aim}

The objective of this research was to study the effect of SLIT on HDM sensitized patients with AD in a randomized controlled trial.

\section{Material and methods \\ Study plan \\ This is a randomized controlled study with two paral- lel groups. The patients included in the trial had to be AD}

Address for correspondence: Na Lu, Department of Dermatology, Affiliated Shunde Hospital of Guangzhou Medical University, China, e-mail: luna_sd@163.com Received: 5.07 .2019 , accepted: 13.07 .2019$. 
patients over the age of 4 years, with a Scoring Atopic Dermatitis (SCORAD) score between 7 and 40, and no persistent bronchial asthma or food allergies. They were randomly divided into SLIT or control groups. Patients in the SLIT group were treated with Dermatophagoides farinae (Der.f.) drops and necessary symptomatic drugs. Patients in the control group could only be treated with symptomatic drugs. The treatment period was 2 years. During the treatment, the SCORAD and a visual analog scale (VAS) score were recorded at regular intervals. A serum level of Der.f.-specific IgE was tested at the baseline and after 24-month treatment. The type and amount of symptomatic drug used was recorded throughout the trial period, and so was the occurrence of adverse events.

All procedures performed in studies involving human participants were in accordance with the ethical standards of the institutional and/or national committee and with the 1975 Helsinki Declaration and its later amendments or comparable ethical standards. Informed consent was obtained from all individual participants included in the study.

\section{Patients}

\section{Patients of both sexes, aged between 4 and 60 years, were enrolled.}

Inclusion criteria were the following: (i) clinical history of chronic AD of over 2 years; (ii) the level of serum Der.f.-specific IgE is class 2 or above (ImmunoCAP; Phadia, Uppsala, Sweden); (iii) during pollen season, no symptom exacerbation was reported; (iv) SCORAD scores greater than 7 but smaller than 40 or less [11].

Exclusion criteria were as follows: (i) bronchial asthma requiring regular treatment with inhaled steroids; (ii) forced expiratory volume in $1 \mathrm{~s} \leq 70 \%$ of predicted value; (iii) history of actual persistent food allergy; (iv) any previous course of immunotherapy; (v) severe systemic disorders (e.g., cystic fibrosis, diabetes, celiac disease) or malignancies; and (iv) patients who received intercurrent treatment with $\beta$-blockers or ACEI during the previous 6 months.

\section{Serum slgE tests}

Serum slgE tests were performed according to recommendations by using a panel of biologically standardized allergens (ImmunoCAP; Phadia, Uppsala, Sweden) which included house dust mite (Dermatophagoides pteronyssinus, Der.p.; Der.f.), 5-grass mixture (Ambrosia artemisiifolia, Artemisia argyi, Argyranthemum frutescens, Taraxacum mongolicum, Solidaginis Herba), mixed tree, Cladosporium species, and dog and cat allergens.

\section{Treatments}

In the control group, treatment with only oral levocetirizine hydrochloride tablets and topical fluticasone propionate are permitted.
Patients in the treatment group were treated with Der.f. drops (CHANLLERGEN, Wolwo Bio-Pharmaceutical Company, Zhejiang, China) for 24 months. According to instructions of the manufacturer of Der.f. drops, the biologically standardized extracts were labelled with the concentration of total Der.f.

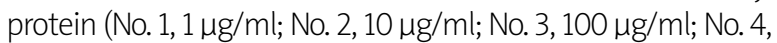
$333 \mu \mathrm{g} / \mathrm{ml}$; No. 5, $1000 \mu \mathrm{g} / \mathrm{ml})$. In the first 4 weeks of SLIT, patients were administered increasing doses starting from Der.f. drops No. 1 to No. 4, i.e., D. farinae drops No. 1 were used in the first week, Der.f. drops No. 2 in the second week and Der.f. drops No. 3 in the third week, of those were given respectively as $1,2,3,4,6,8$, and 10 drops (each drop of $40 \mu \mathrm{l}$ ) day after day in a week. For patients aged 4-14 years, maintenance therapy with three drops of Der.f. drop No. 4 per day from the fourth week to the $24^{\text {th }}$ month. For patients older than 14 years, Der.f. drops No. 4 three drops a day were given in the fourth and the fifth week and then the maintenance therapy with two drops of Der.f. drop No. 5 per day followed from the sixth week to the $24^{\text {th }}$ month. Patients were instructed to keep the Der.f. drops under the tongue for 1-3 min and then swallow. The first dose of Der.f. drops was administered in the physician's office with specific instructions, and after $30 \mathrm{~min}$ observation, patients could leave the hospital. The step-up dosage protocol was standardized as described [12]. Regular oral levocetirizine hydrochloride tablets and topical fluticasone propionate as the control group were added depending on individual diseases.

In the case of cutaneous superinfection, the physician could prescribe a 6-day course of clarithromycin (15 mg/ $\mathrm{kg} /$ day). No other treatment, including moisturizers, was allowed during the study.

\section{Outcome measures}

\section{Patients' compliance}

Patients' reasons and rate of withdrawal were analysed, and participants failing to complete treatment for 24 months were excluded from the study. The higher the withdrawal rate is, the lower the compliance.

\section{SCORAD score}

All the patients were followed up with regular clinic visits during the whole study. The change in SCORAD versus baseline was the primary outcome. The baseline SCORAD was assessed before randomization (run-in period of 1 month) and then after 6, 12, and 24 months of treatment. A $\Delta$ SCORAD (difference from baseline) was calculated for analysis.

\section{VAS score}

At each visit, patients were asked to mark a line on the VAS. They were asked to quantify the overall AD symptoms on a VAS ranging from 0 (no symptoms at all) to 10 (very severe symptoms). To fill the VAS, they had to answer the question "How was the eczema in the last month?". As a tool for subjective evaluation, it reflects the quality of life of patients. 


\section{Rescue medications score}

The patients were required to record drugs' use in the diary card. The use of medications was scored 1 point for each dose of oral levocetirizine hydrochloride tablets or topical fluticasone propionate and six points for every 6-day course of clarithromycin. The latter was given only in the case of superinfection.

\section{Level of serum specific IgE to Der.f.}

Levels of specific lgE to Der.f. were measured at baseline and after 24 months of SLIT. It was measured by ImmunoCAP (Phadia, Uppsala, Sweden), and expressed in kU/l.

\section{Statistical analysis}

Statistical analysis was carried out using SPSS 20.0 software (IBM Corp., Armonk, NY). All tests were 2-tailed, and the level of significance was set at 0.05. The analysis of variance (ANOVA) or Friedman test was used for intragroup comparison (each visit versus baseline). The t-test or Wilcoxon test was used to examine the difference between the treatment group and the control group.

\section{Results}

\section{Population characteristics}

Ninety-six patients, aged between 4 and 60 years (mean age: 26.5 years; 47 males) were enrolled in the
Table 1. Demography and clinical characteristics of the enrolled patients at baseline

\begin{tabular}{lcc}
\hline Parameter & SLIT group & $\begin{array}{c}\text { Control } \\
\text { group }\end{array}$ \\
\hline Patients, $n$ & 48 & 48 \\
\hline Age [years] & $27.06 \pm 4.9$ & $26.53 \pm 4.1$ \\
\hline Men/women, ratio & $23 / 48$ & $24 / 48$ \\
\hline Duration of AD [years] & $5.41 \pm 3.3$ & $5.18 \pm 3.8$ \\
\hline SCORAD score & $25 \pm 6$ & $26 \pm 7$ \\
\hline Patients with asthma, $n(\%)$ & $4(8.3)$ & $5(10.4)$ \\
\hline Patients with rhinitis, $n$ (\%) & $7(14.6)$ & $8(16.7)$ \\
\hline Specific lgE to Der.f., means \pm SDs [kU/l] & $4.42 \pm 2.04$ & $4.60 \pm 2.33$
\end{tabular}

study and randomized so that 48 patients were allocated to the SLIT group and the remaining to the control group. All patients had serum sensitization to dust mite. There were no significant differences between the two groups in age, sex ratio, duration, severity, slgE level at baseline (all $p>0.05$; Table 1 ). $81.3 \%$ of the patients (39/48) in the treatment group and $79.2 \%$ of the patients (38/48) in the control group had completed the study $(p>0.05)$. No patients withdrew from the study because of AEs (Figure 1).

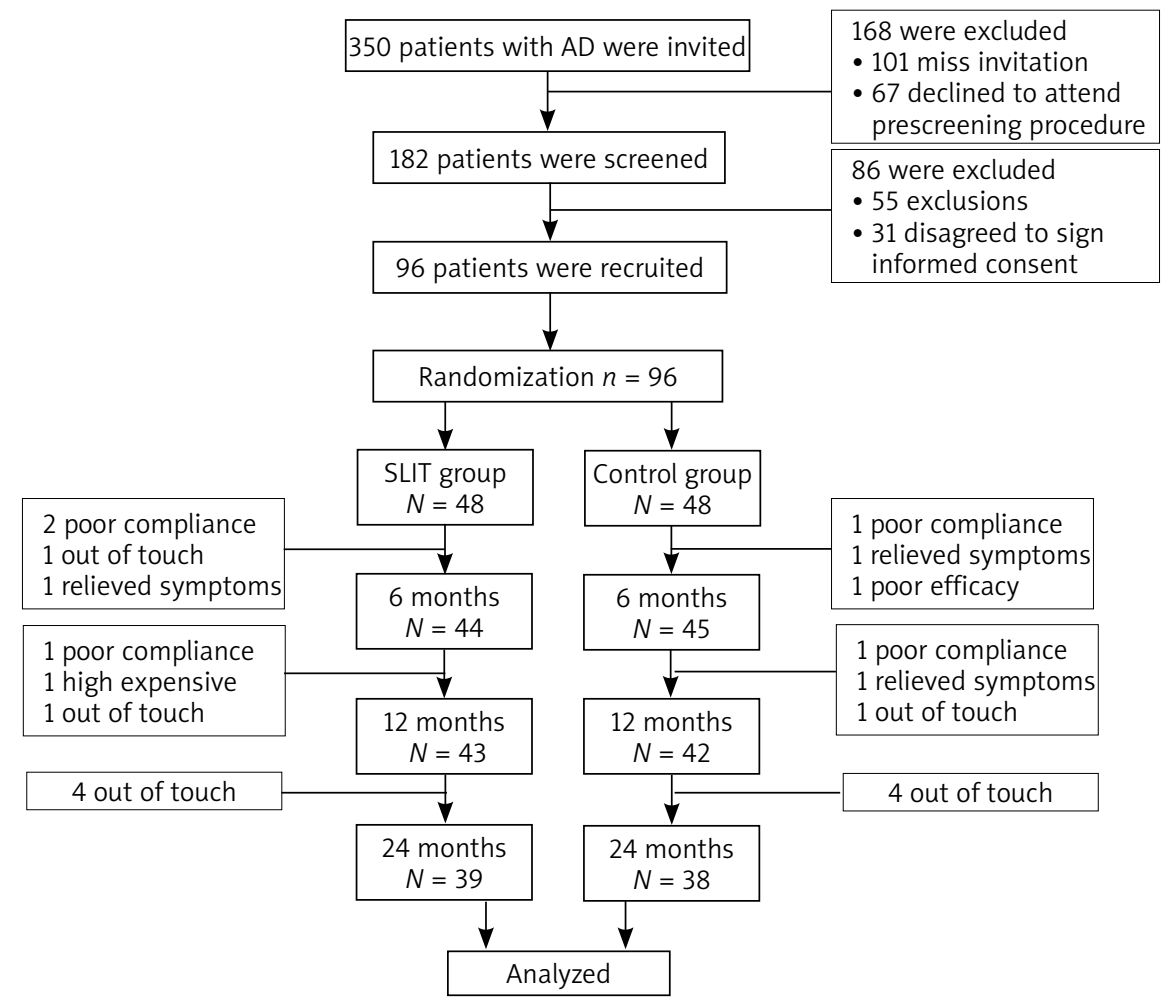

Figure 1. Flowchart and dropout analysis 


\section{SCORAD score}

In the SLIT group, the mean SCORAD score decreased from baseline to any post-baseline time point, but in the control group, there is no substantial decrease at 12 and 24 months, as shown in Figure 2. The comparison between groups of changes from baseline revealed statistically significant differences in favour of the SLIT group at month $12(p<0.05)$ and at month $24(p<0.05)$, whereas the difference between groups at month 6 was not statistically significant. The mean (SD) change from baseline to the final visit (month 24) was -10.02 (4.43) in the SLIT group and -2.86 (2.57) in the control group.

\section{VAS score}

The VAS score of SLIT significantly decreased in the SLIT group compared with the baseline $(p<0.05)$ and control group ( $p<0.05)$ from month 12 , whereas no change versus baseline was observed in the placebo group (all $p>0.05$ ). At the end of the study, an overall increase versus baseline $(+2.2 \%)$ in the control group and a decrease in the active group $(-45.7 \%$; $p<0.01)$ was observed, respectively (Figure 3 ).

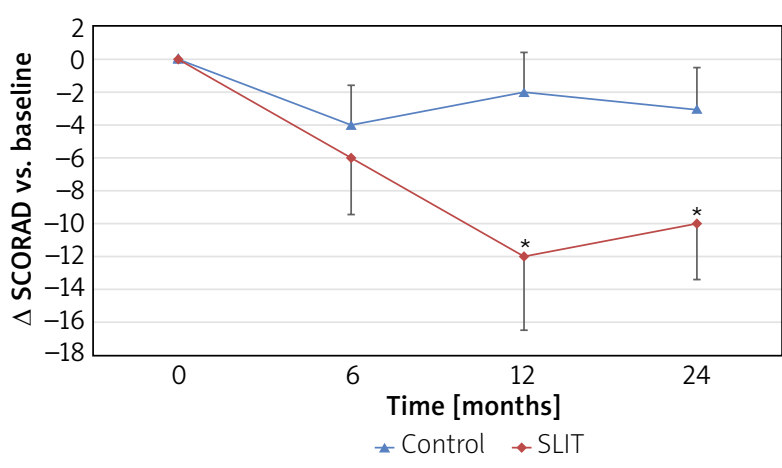

Figure 2. Change from baseline ( $\triangle$ SCORAD) in the SLIT and control groups at different time points. ${ }^{*}$ Significant differences between groups

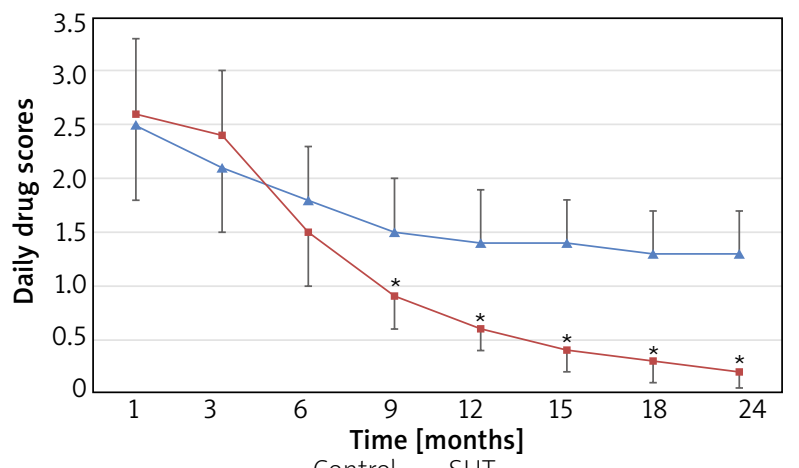

Figure 4. Daily drug scores at different time points. *Significant differences between groups

\section{Rescue medications score}

The average daily rescue medications scores were compared at different time points. The SLIT group was significantly lower compared with the control group from month 12 (all $p<0.05$ ). Moreover, at 24-month followup, average daily $\Delta$ drug scores showed a clear reduction (take the first month as the baseline) in the SLIT group ( $\Delta$ drug scores, -2.4$)$ compared with the control group $(\Delta$ drug scores, -1.2$)(p<0.01)$ (Figure 4$)$.

\section{Serum Der.f.-specific slgE level}

There were no significant differences in serum Der.f.specific slgE level between the SLIT and the control group after 24 months ( $p>0.05$ ) (Figure 5).

\section{Safety}

No patients required hospitalization or withdrew from the study because of AEs. No severe systemic AEs, anaphylaxis, acute attack of asthma, or use of adrenaline were reported. Three patients in the treatment group reported five AEs (3 transient oral itching and 2 gastroin-

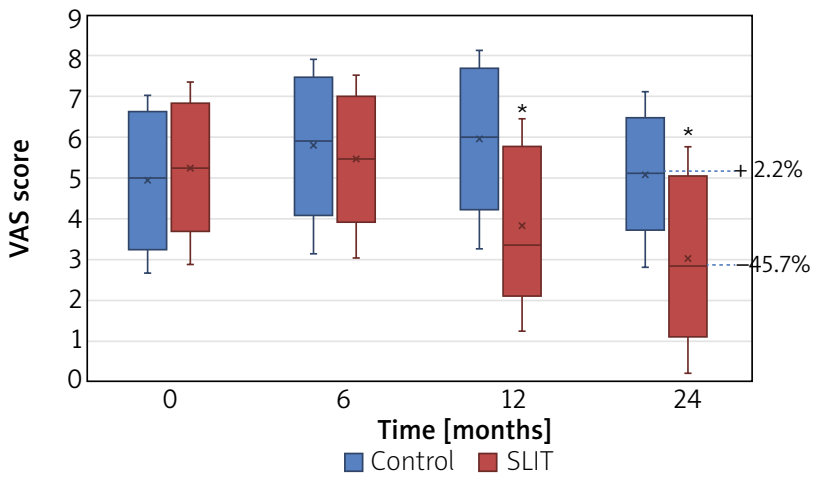

Figure 3. VAS scores (median, range) in the SLIT and control groups at different time points. The box plot indicates the range, median and quartiles. *Significant differences between groups

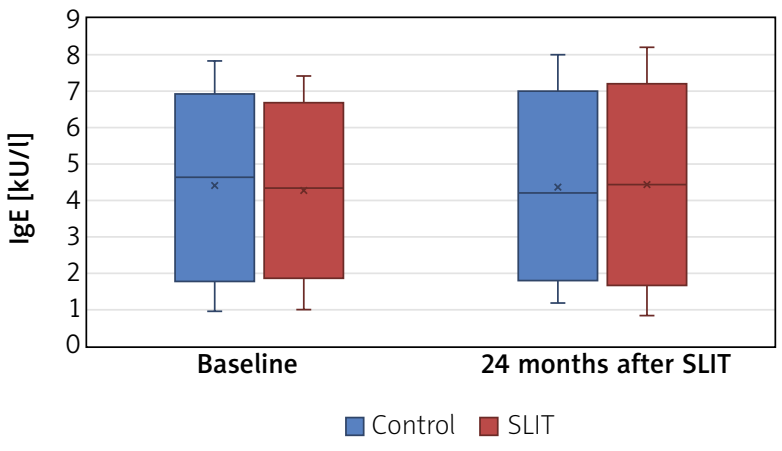

Figure 5. Changes of specific IgE serum levels at baseline and after 24 months of SLIT treatment. The box plot indicates the range, median and quartiles 
testinal discomfort), and 2 patients in the control group reported two AEs (1 headache and 1 gastrointestinal discomfort). Most of the AEs were grade 1 and were relieved within a week with or without medication.

\section{Discussion}

Atopic dermatitis is a common chronic inflammatory skin disease, with a worldwide increase in prevalence, bringing a great impact on the life of patients [13, 14]. The pathogenesis of $A D$ is not yet clear and it is currently believed that $A D$ was related to genetics, environment and lifestyle [15]. Allergens, such as HDM, as an important factor in the environment, promote the onset of $A D[16$, 17]. From this perspective, SIT may bring some benefits to the treatment of AD.

The role of SCIT in the treatment of AD has been confirmed by several studies. In a recent study, the researchers observed the effect of HDM extract SCIT on patients with HDM-sensitive AD, which showed reduced SCORAD scores and reduced demand for topical corticosteroids [5]. However, the clinical use of SCIT has been limited by site restrictions and safety concerns. SLIT is a safer and more convenient alternative to SCIT, but so far it has limited use in the clinical treatment of AD patients [18]. Recently, only a small number of studies have reported the results of SLIT in patients with AD [8-10, 19, 20]. Our objective was to evaluate the clinical efficacy of SLIT in adult and paediatric patients with AD. The results revealed significant decreases in SCORAD scores and drug intake after SLIT treatment. VAS score also showed an improved trend in the SLIT treatment group, with a significant difference compared with the control group after 12 months of treatment. This is similar to results reported in previous studies.

Currently, there are no recognized serological indicators for the prediction and determination of the efficacy of SLIT in AD [21]. However, some researchers have studied changes in IgE levels before and after SIT treatment, and the results have not been consistent $[5,19,22]$. In our study, levels of allergen-specific IgE in both groups remained unchanged before and after treatment. Further studies may be needed to determine the effects of specific immunotherapy on IgE levels.

SLIT's safety has been widely acknowledged [23, 24]. The most common adverse reactions are local side effects, including numbness, itching, swelling of the mouth and tongue. In our study, only 3 patients developed transient oral itching or gastrointestinal discomfort, and all were relieved within a week with or without medication. Moreover, no serious systemic reactions or anaphylaxis was observed during the study.

This is the first study of SLIT involving both children and adults with AD in China. Although it shows that SLIT with HDM extracts is effective and tolerable in Chinese children and adult patients with $A D$, there are several limitations in this study. Firstly, the patients in both groups took medications on-demand, which results in symptom improvement in the control group. In addition, the sample size is small and the observation duration was not long enough. In the future, a large number of confirmatory controlled studies and long-term trials are required in order to verify the effect and safety of SLIT in Chinese patients with AD.

\section{Conclusions}

This randomized controlled study has suggested that SLIT to HDM allergen extract could generate significant clinical efficacy for AD patients, as shown by the significant overall reduction in SCORAD scores and the need for rescue medicine. In the future, a large number of confirmatory controlled studies with long-term course are required to reinforce these current results in China.

\section{Acknowledgments}

We thank the patients for their availability in taking part in this trial and for the excellent cooperation they have shown.

\section{Conflict of interest}

The authors declare no conflict of interest.

\section{References}

1. Adkis C, Akdis M, Bieber T. Diagnosis and treatment of atopic dermatitis in children and adults: European Academy of Allergology and Clinical Immunonology/American Academy of Allergy, Asthma and Immunology/PRACTALL consensus report. J Allergy Clin Immunol 2006; 118: 152-69.

2. Johansson SG, Bieber T, Dahl R, et al. Revised nomenclature for allergy for global use: report of the Nomenclature Review Committee of the World Allergy Organization, October 2003. J Allergy Clin Immunol 2004; 113: 832-6.

3. Scalabrin DM, Bavbek S, Perzanowski MS, et al. Use of specific IgE in assessing the relevance of fungal and dust mite allergens to atopic dermatitis: a comparison with asthmatic and nonasthmatic control subjects. J Allergy Clin Immunol 1999; 104: 1273-9.

4. Till SJ, Francis JN, Nouri-Aria K, Durham SR. Mechanisms of immunotherapy. J Allergy Clin Immunol 2004; 113: 1025-34.

5. Werfel T, Breuer K, Ruéff F, et al. Usefulness of specific immunotherapy in patients with atopic dermatitis and allergic sensitization to house dust mites: a multi-centre, randomized, dose-response study. Allergy 2006; 61: 202-5.

6. Nahm DH, Kim ME. Treatment of severe atopic dermatitis with a combination of subcutaneous allergen immunotherapy and cyclosporin. Yonsei Med J 2012; 53: 158-63.

7. Canonica GW, Cox L, Pawankar R, et al. Sublingual immunotherapy: World Allergy Organization Position Paper 2013 update. World Allergy Organ J 2014; 7: 6.

8. Pajno GB, Caminiti L, Vita D, et al. Sublingual immunotherapy in mite-sensitized children with atopic dermatitis: a randomized, double-blind, placebo-controlled study. J Allergy Clin Immunol 2007; 120: 164-70. 
9. Qin YE, Mao JR, Sang YC, et al. Clinical efficacy and compliance of sublingual immunotherapy with Dermatophagoides farinae drops in patients with atopic dermatitis. Int I Dermatol 2014; 53: 650-5.

10. Hyang-Suk Y, Min-Young Y, Gun-Wook K, et al. Effectiveness of specific sublingual immunotherapy in Korean patients with atopic dermatitis. Ann Dermatol 2017; 29: 1-5.

11. Stalder JF, Taieb A, Atherton DJ. Severity scoring of atopic dermatitis: the SCORAD Index. Dermatology 1993; 186: 23-31.

12. Wang DH, Chen L, Cheng L, et al. Fast onset of action of sublingual immunotherapy in house dust mite induced allergic rhinitis. A multicenter, randomized, double-blind, placebocontrolled trial. Laryngoscope 2013; 123: 1334-40.

13. Asher MI, Montefort S, Bjorksten B, et al. ISAAC Phase Three Study Group. Worldwide time trends in the prevalence of symptoms of asthma, allergic rhinoconjunctivitis, and eczema in childhood: ISAAC Phases One and Three repeat multicountry cross-sectional surveys. Lancet 2006; 368: 733-43.

14. Schram ME, Tedja AM, Spijker R, et al. Is there a rural/urban gradient in the prevalence of eczema? A systematic review. Br J Dermatol 2010; 162: 964-73.

15. Cork MJ, Robinson DA, Vasilopoulos Y, et al. New perspectives on epidermal barrier dysfunction in atopic dermatitis: gene-environment interactions. J Allergy Clin Immunol 2006; 118: 3-21.

16. Fuiano N, Incorvaia C. Dissecting the causes of atopic dermatitis in children: less foods, more mites. Allergol Int 2012; 61: 231-43.

17. Hostetler SG, Kaffenberger B, Hostetler T, Zirwas MJ. The role of airborne proteins in atopic dermatitis. J Clin Aesthet Dermatol 2010; 3: 22-31.

18. Durham S. Tradition and innovation: finding the right balance. J Allergy Clin Immunol 2007; 119: 792-5.

19. Cadario G, Galluccio AG, Pezza M, et al. Sublingual immunotherapy efficacy in patients with atopic dermatitis and house dust mites sensitivity: a prospective pilot study. Curr Med Res Opin 2007; 23: 2503-6.

20. Di Rienzo V, Cadario G, Grieco T, et al. Sublingual immunotherapy in mitesensitized children with atopic dermatitis: a randomized, open, parallel-group study. Ann Allergy Asthma Immunol 2014; 113: 671-3.e1.

21. Novak N. Allergen specific immunotherapy for atopic dermatitis. Curr Opin Allergy Clin Immunol 2007; 7: 542-6.

22. Silny W, Czarnecka-Operacz M. Specific immunotherapy in the treatment of patients with atopic dermatitis: results of double blind placebo controlled study. Pol Merkur Lekarski 2006; 21: 558-65.

23. Cox LS, Larenas Linnemann D, Nolte H, et al. Sublingual immunotherapy: a comprehensive review. J Allergy Clin Immunol 2006; 117: 1021-35.

24. Gómez Vera J, Flores Sandoval G, Orea Solano M, et al. Safety and efficacy of specific sublingual immunotherapy in patients with asthma and allergy to Dermatophagoides pteronyssinus. Rev Alerg Mex 2005; 52: 231-6. 